\title{
苔藓-蓝藻共生体关系与固氮能力研究进展
}

\author{
皮春燕 ${ }^{1,2}$ 刘 金金 王吉 $^{3}$ 包维楷 ${ }^{*}$
}

${ }^{1}$ 中国科学院山地生态恢复与生物资源利用重点实验室, 生态恢复与生物多样性保育四川省重点实验室, 中国科学院成都生物研究所, 成都 $610041 ;$

${ }^{2}$ 中国科学院大学, 北京 $100049 ;{ }^{3}$ 上海师范大学生命与环境科学学院, 上海 200234

摘 要 苔藓-蓝藻共生体(BCS)能固氮, 是养分贫㾑地区森林氮输入的不可忽视的来源。BCS关系与固氮能力研究为科学认 识生态系统氮输入与氮循环过程和机理提供了新的视角和有效途径, 具有重要的理论价值。然而, BCS关系、固氮作用与机 理的研究迄今未受到足够关注, 报道较少, 认识仍然是零星而片段化的。基于系统查阅的相关文献, 该文综述了 BCS的种类 组成与共生关系类型、固氮能力及所固定氮的去向及其影响因素和作用机理, 指出了存在的问题及需要深入关注和亟待突破 的 4 个研究方向。

关键词＼cjkstart苔藓-蓝藻共生体; 固氮作用; 生态系统氮输入; 氮循环; 影响因素

皮春燕, 刘金金, 王喆, 包维楷 (2018). 苔藓-蓝藻共生体关系与固氮能力研究进展. 植物生态学报, 42, 407-418. DOI: 10.17521/cjpe.2017.0191

\section{Bryophyte-cyanobacteria symbioses and their nitrogen fixation capacity-A review}

PI Chun-Yan ${ }^{1,2}$, LIU Xin ${ }^{1}$, WANG Zhe ${ }^{3}$, and BAO Wei-Kai ${ }^{{ }^{*}}$

${ }^{1}$ Key Laboratory of Mountain Ecological Restoration and Bioresource Utilization \& Ecological Restoration Biodiversity Conservation Key Laboratory of Sichuan Province, Chengdu Institute of Biology, Chinese Academy of Sciences, Chengdu 610041, China; ${ }^{2}$ University of Chinese Academy of Sciences, Beijing 100049, China; and ${ }^{3}$ College of Life and Environmental Sciences, Shanghai Normal University, Shanghai 200234, China

\begin{abstract}
Bryophyte-cyanobacteria symbiosis (BCS) is a key source of nitrogen input into ecosystems in nutrient-poor regions. Investigating BCS relationships and the nitrogen fixation capacity can be a new pathway and window to explore the process and mechanism of nitrogen input and nitrogen cycling. However, BCS relationships and nitrogen fixation/cycling processes and mechanisms remain poorly studied, and most of these studies have only focused on the boreal forest, with no report from Chinese forests. Based on systematic literature search and analysis, this review provides a summary on BCS relationships, the nitrogen fixation capability of BCS, the fate of fixed nitrogen, as well as the environmental factors and driving mechanisms of BCS. Firstly, we synthesized different types of BCS, the mechanisms by which the fixed nitrogen is transferred to and used by other plants within the forest, the rate of fixed nitrogen, the factors influencing the rate of nitrogen fixation. Moreover we point out the existing problems that need to pay close attention to and at least four research directions need to break through. Furthermore, the theoretical basis of BCS is provided for further research, promote and deepen the cognition of BCS and nitrogen-fixing research.
\end{abstract}

Key words bryophyte-cyanobacteria symbiosis; nitrogen fixation; ecosystem nitrogen inputs; nitrogen cycle; driving factor

Pi CY, Liu X, Wang Z, Bao WK (2018). Bryophyte-cyanobacteria symbioses and their nitrogen fixation capacity-A review. Chinese Journal of Plant Ecology, 42, 407-418. DOI: 10.17521/cjpe.2017.0191

生态系统净初级生产力常常受到可利用氮的限 制(Tamm, 1991)。生物固氮是自然生态系统最主要 的氮来源(Stewart, 1966; Cleveland et al., 1999), 但 在许多生态系统, 如分布在高海拔和高纬度地区的 亚高山针叶林和北方针叶林中, 往往缺乏能够与固 氮根瘤菌形成共生关系的物种(如豆科植物), 那么
生态系统的氮来源问题究竟是如何解决的呢? 后来 发现以蓝藻类(或蓝细菌, cyanobacteria)为主的固氮 微生物是这些生态系统中氮的主要来源(Deluca, 2002b; Reed et al., 2011)。蓝藻是一类自养原核生物 (Rai et al., 2000), 能够独立营自养生存, 也能以附 生(epiphyte)或内生(endophyte/entophyte)等形式与 
真菌和苔藓等形成共生体(Meeks, 1990, 2007; Rai et al., 2000; Adams \& Duggan, 2008), 其生物固氮作用 主要靠固氮酶进行, 且固氮酶集中于异形胞 (heterocyst)中, 所以氮固定常发生在蓝藻体内的异 形胞中, 而异形胞的数量与蓝藻生境有密切关系 (Adams \& Duggan, 2008)。

苔藓植物是亚高山针叶林和北方针叶林林下的 主要层片(图1), 盖度达60\%以上(刘俊华等, 2005; Liu \& Bao, 2014), 生物量可达 $3 \mathrm{t} \cdot \mathrm{hm}^{-2}$ 以上, 占森林 总初级生产量的1/3 (Deluca et al., 2002b)。林下苔藓 层在调节微气候方面发挥着重要作用, 直接或间接 地影响森林生态系统中养分元素的生物地球化学循 环过程(Cornelissen et al., 2007)。其中一个有趣的但 目前所知甚少的途径就是苔藓能够主动释放特定化 学物质促进蓝藻与其形成共生关系(Bay et al., 2013), 苔藓 - 蓝藻共生体 (bryophyte-cyanobacteria symbiosis)能在森林地表进行更高效的氮固定和氮 转运。已发现得益于苔藓供给碳水化合物等营养物 质及苔藓层所提供的相对稳定的温度、水分、湿度 等微环境条件, 苔藓-蓝藻类共生体中的蓝藻异形 胞数量与固氮能力均显著高于独立营自养生存的蓝 藻。比如, 共生体中蓝藻体内异形胞数量可达到独 立生存时的 6-10倍, 固氮速率则可增加至 7倍 (Adams \& Duggan, 2008), 另外也发现共生体固定 的氮转移至苔藓植物可使苔藓植物生物量增加 (Berg et al., 2012)。苔藓-蓝藻共生体在北方森林和 极地环境中广泛存在(Zackrisson et al., 2004), 由于 其巨大的生物量, 林下苔藓-蓝藻共生体在亚高山 针叶林和北方针叶林生态系统中的固氮速率可达
1-2 $\mathrm{kg} \cdot \mathrm{hm}^{-2} \cdot \mathrm{a}^{-1}$, 而后通过调落物分解、淋溶等形式 释放到外界环境中, 被其他植物利用, 是森林生态 系统重要的氮汇和氮源(Wilson \& Coxson, 1999; Deluca et al, 2002b; Gerber et al., 2010; Gundale et al, 2011), 对各生态系统乃至全球氮输入与氮循环具 有不容忽视的生态学意义。因此, 系统深入认识苔 藓-蓝藻类共生体关系及其固氮效应与机制对于揭 示森林养分循环过程与森林管理具有重要的理论 价值。

然而苔藓-蓝藻共生体关系及效应的相关研究 目前仍然贫乏, 已有认识还处于零散和片段化状态, 这一显著影响森林生态系统氮循环的关键途径及其 过程仍未引起人们的广泛兴趣和重视。因此, 系统 地分析、梳理和归纳已有对苔藓-蓝藻共生体固氮研 究的进展和科学认识是十分必要的。本文作者系统 查阅了过去 50 年来苔藓-蓝藻共生体及其相关研究 的文献报道, 试图综述如下方面的研究现状：(1)苔 藓-蓝藻共生体的类群、物种组成与共生关系; (2)苔 藓-蓝藻共生体的固氮能力及其所固定氮的去向; (3)影响共生体关系及其固氮能力的因素与作用机 理; 进一步梳理出当前已有的结论与需要聚焦的方 向, 为苔藓-蓝藻共生体关系及其效应的深入研究 提供基础。

\section{1 苔藓-蓝藻共生体类群及共生关系}

\section{1 苔藓-蓝藻共生体类群与组成物种}

从现有报道来看, 依据苔藓类群可将苔藓-蓝 藻共生体分为 3 类, 即角苔-蓝藻共生体 (hornwortcyanobacteria symbiosis)、苔-蓝藻共生体(liverwort-

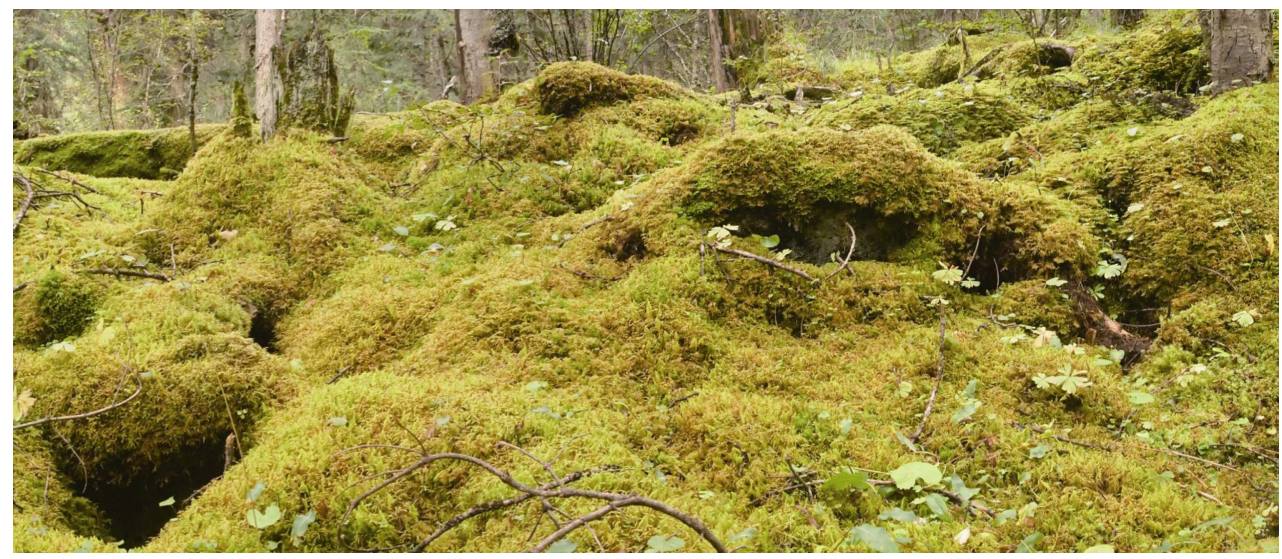

图1 青藏高原东部亚高山冷杉老龄林林下苔藓层片。苔藓层主要由塔藓、锦丝藓、毛梳藓和赤茎藓等构成。

Fig. 1 An old-growth Abies fargesii var. faxoniana forest site in eastern Qinghai-Xizang Plateau with a moss dominated understory. Dominate moss species are Hylocomium splendens, Actinothuidium hookeri, Ptilium crista-castrensis, and Pleurozium schreberi.

www.plant-ecology.com 
cyanobacteria symbiosis) 和藓 - 蓝藻共生体 (mosscyanobacteria symbiosis)。迄今已知能与蓝藻共生固 氮的苔藓共 35 属 41 种(表 1$)$, 在全球已知苔藓种类中 仅占很小比例。

角苔类在角苔属(Anthoceros)、树角苔属(Dendroceros)、短角苔属(Notothylas)和黄角苔属(Phaeoceros) 4 个属的物种与蓝藻有共生固氮关系 (Meeks, 1990), 但尚未明确鉴定到具体种类; 发现 苔类中有 6 属的物种能够与蓝藻共生, 其中仅 2 属明 确至种, 即Anastrophyllum involutifolium 和Chiloscyphus leptanthus (Rippka et al., 1979; Meeks, 1990;
West \& Adams, 1997; Houle et al., 2006; ArrónizCrespo et al., 2014)。

目前发现能与蓝藻共生固氮的藓类种类相对而 言最多, 有25属31种。其中仍有5属(真藓属(Bryum)、 镰刀藓属(Drepanocladus)、Dupontia、紫䓵藓属 (Grimmia)和毛梳藓(Ptilium))未见明确的物种报道; 仅4属(黑藓属(Andreaea)、砂藓属(Racomitrium)、泥 炭藓属(Sphagnum)和镰刀藓属(Drepanocladus))有 1 个以上的物种能够与蓝藻共生, 其中已在砂藓属的 4 个种中发现共生关系, 分别是Racomitrium subcrispipilum、R. laevigatum、 $R$. lanuginosum和 $R$.

表1＼cjkstart苔蘚-蓝藻共生体类型及共生形式

Table 1 Types and relationships of bryophyte-cyanobacteria symbiosis

\begin{tabular}{|c|c|c|c|}
\hline 类型 Type & $\begin{array}{l}\text { 苔蘚种类及与蓝藻关系 } \\
\text { Bbryophyte and the relationship with } \\
\text { cyanobacteria (En or Ep) }\end{array}$ & 蓝藻种类 Cyanobacteria & 参考文献 Reference \\
\hline $\begin{array}{l}\text { 角苔-藻共生体 } \\
\text { Hornwort-cyanobacteria } \\
\text { symbiosis }\end{array}$ & $\begin{array}{l}\text { 角苔属 Anthoceros sp. (En 1) } \\
\text { 树角苔属 Dendroceros sp. (En 1) } \\
\text { 短角苔属 Notothylas sp. (En 1) } \\
\text { 黄角苔属 Phaeoceros sp. (En 1) }\end{array}$ & 1 念珠藻属 Nostoc sp. & $\begin{array}{l}\text { Rippka et al., 1979; Meeks, 1990; West } \\
\text { \& Adams, 1997; Houle et al., 2006; } \\
\text { Arróniz-Crespo et al., } 2014\end{array}$ \\
\hline $\begin{array}{l}\text { 苔-藻共生体 } \\
\text { Liverwort-cyanobacteria } \\
\text { symbiosis }\end{array}$ & $\begin{array}{l}\text { Anastrophyllum involutifolium (Ep1) } \\
\text { 壶苞苔属 Blasia sp. (Ep 1, 2, 4; En 1) } \\
\text { Cavicularia sp. (En 1) } \\
\text { Chiloscyphus leptanthus (Ep 1) } \\
\text { 地钱属 Marchantia sp. (Ep 1) } \\
\text { 光䓯苔 Porella sp. (Ep 1) }\end{array}$ & $\begin{array}{l}1 \text { 念珠藻属 Nostoc sp. } \\
2 \text { 眉藻属 Calothrix sp. } \\
3 \text { 真枝藻属 Stigonema sp. } \\
4 \text { Chlorogloeopsis sp. }\end{array}$ & $\begin{array}{l}\text { Rippka et al., 1979; Meeks, 1990; West } \\
\text { \& Adams, 1997; Houle et al., 2006; } \\
\text { Arróniz-Crespo et al., } 2014\end{array}$ \\
\hline $\begin{array}{l}\text { 藓-藻共生体 } \\
\text { Moss-cyanobacteria } \\
\text { symbiosis }\end{array}$ & 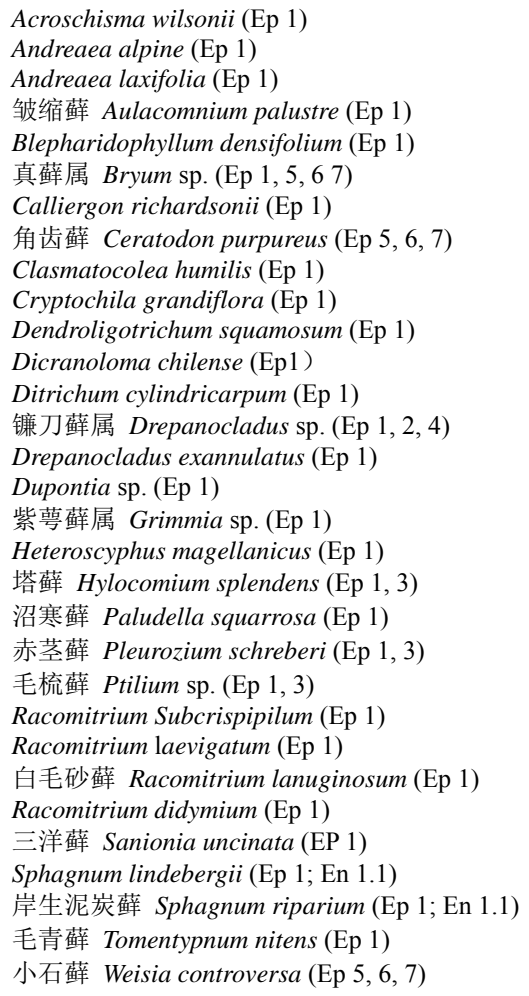 & $\begin{array}{l}1 \text { 念珠藻属 Nostoc sp. } \\
1.1 \text { 灰色念珠藻 Nostoc muscorum } \\
2 \text { 眉藻属 Calothrix sp. } \\
3 \text { 真枝藻属 Stigonema sp. } \\
4 \text { 伪枝藻属 Scytonema sp. } \\
5 \text { Anobena sp. } \\
6 \text { 颤藻属Oscillatoria sp. } \\
7 \text { 鞘丝藻属 Lyngbya sp. }\end{array}$ & $\begin{array}{l}\text { Granhall \& Selander, 1973b; Rippka et } \\
\text { al., 1979; Reddy \& Giddens, 1981; } \\
\text { Davey \& Marchant, 1983; Meeks, } \\
\text { 1990; West \& Adams, 1997; Gentili et } \\
\text { al., 2005; Houle et al., 2006; Gavazov } \\
\text { et al., 2010; Arróniz-Crespo et al., } 2014\end{array}$ \\
\hline 小计 Total & 41 & 8 & \\
\hline
\end{tabular}

En, 内生; Ep, 附生; 数字表示与苔藓共生的蓝藻种类, 如Anthoceros sp. (En 1)表示藻类Nostoc sp.内生于角苔Anthoceros sp.中。

En, entophyte/endophyte; Ep, epiphyte; The number indicates to which cyanobacteria the bryophyte was symbiosised with, for example, "Anthoceros sp. (En 1)" means the cyanobacteria, and "Nostoc sp." is entophytically associated with the hornwort "Anthoceros sp.". 
didymium。在亚高山针叶林和北方针叶林林下常见 的藓类塔藓(Hylocomium splendens)和赤茎藓(Pleurozium schreberi)均具有与蓝藻共生固氮的能力, 但 在波叶曲尾藓(Dicranum polysetum) 和金发藓( $\mathrm{Pol}-$ ytrichum commune)中却未发现共生固氮现象(Bay et al., 2013)。

目前已知能与苔藓形成共生关系的固氮蓝藻有 8属(表1)。其中, 除念珠藻属(Nostoc)的灰色念珠藻 (Nostoc muscorum) 外, 其他7个属未明确鉴定到具 体种类(Granhall \& Selander, 1973; Rippka et al., 1979; Reddy \& Giddens, 1981; Davey \& Marchant, 1983; West \& Adams, 1997; Houle et al., 2006; ArrónizCrespo et al., 2014)。

\section{2 苔藓-蓝藻共生体的共生关系类型}

苔藓和蓝藻的共生关系有 2 种: 蓝藻内生和附 生于苔藓。角苔与蓝藻共生体均为内生型关系。在 苔-蓝藻共生关系中, 蓝藻的内生位置至少有 2 种报 道。一种是有关念珠藻属(Nostoc)物种生长于苔类配 子体的耳廓(auricles)中(Rippka et al., 1979; Meeks, 1990; West \& Adams, 1997; Houle et al., 2006), 另一 种是生长于配子体腹面的黏液腔(slime cavities)中 (Meeks, 1990), 如壶苍苔属(Blasia)和Cavicularia属 能够与念珠藻形成的内生关系。内生型的藓-蓝藻共 生体目前仅发现泥炭藓属中的Sphagnum lindebergii 和岸生泥炭藓(Sphagnum riparium) 与Nostoc muscorum形成的共生体(Granhall \& Selander, 1973)。

附生型的共生关系多见于地钱属(Marchantia)、 光葶苔属(Porella)、裂蔩苔属(Chiloscyphu)、毛叶苔 属(Ptilidium)和挺叶苔属(Anastrophyllum)的苔-藻共 生体以及各种藓-藻共生体中(表1)。除上述泥炭藓属 2种与念珠藻形成内生型的共生外, 其余藓类蓝藻 共生体均为附生形式。其中, Anobena、Oscillatoria 和Lyngbya属的蓝藻可以附生于真藓类角齿藓 (Ceratodon purpureus)和小石藓(Weisia controversa) 中(Reddy \& Giddens, 1981), 念珠藻属蓝藻可以附 生的位置一般在多种藓类的叶片内弯部(Deluca et al., 2002b)。值得注意的是, 藓类在附生型的共生体 中占据主导地位, 而同一苔藓与蓝藻是否能同时具 有内生与附生型共生关系均不清楚。Bay等(2013) 发现, 在氮缺乏时, 分布广泛的赤茎藓和塔藓会释 放特定的引诱剂吸引念珠藻附生, 并促进念珠藻产 生藻殖段和异形胞提高固氮能力; 但当藓类对氮的
需求下降时(例如孢子体被移除时), 共生体的固氮 速率也下降。遗憾的是, 苔藓如何释放特定的引诱 剂及相关作用机制尚未见报道。

\section{2 固氮能力}

苔藓-蓝藻共生固氮能力的定量研究到目前为 止仅聚焦于藓-藻共生体，而缺乏角苔-藻与苔-藻共 生体的报道。归纳已有的报道发现, 各苔藓-蓝藻共 生体的固氮能力介于 $0.25-1.7 \mathrm{~kg} \cdot \mathrm{hm}^{-2} \cdot \mathrm{a}^{-1}$, 如图2所 示, 其中固氮量最高的地区为Deluca等(2002b)报道 的 27 个地区 (瑞典、挪威和芬兰), 固氮量平均值为 $1.7 \mathrm{~kg} \cdot \mathrm{hm}^{-2} \cdot \mathrm{a}^{-1}$; 瑞典的Hornavan湖和Uddjaure湖的 平均固氮量最低, 为 $0.25 \mathrm{~kg} \cdot \mathrm{hm}^{-2} \cdot \mathrm{a}^{-1}$ (Lagerstorm et al., 2007); 我们注意到, 有限的藓-蓝藻共生体的固 氮能力研究主要聚焦在北欧与北美高纬度北方针叶 林或北极圈附近地区 (Rosén \& Lindberg, 1980; Deluca et al., 2008), 其他地区(包括中国)的案例研 究非常贫乏。关于藓-蓝藻共生体的固氮能力动态变 化的报道仅见Deluca等(2002b)的工作，报道了瑞典 Reivo森林中赤茎藓-蓝藻共生体半年(5-11月)的动 态固氮率变化, 发现在苔藓旺盛生长季的6月、8月 底和 10 月出现固氮率峰值, 未见年固氮量动态变化 规律以及其他种类的苔藓-蓝藻共生体固氮能力变 化的报道。

目前报道的苔藓物种仅针对以上区域森林中的 优势种类, 如赤茎藓、塔藓和泥炭藓, 缺少对其他苔 藓种类的研究。就有限案例的比较而言, 赤茎藓-蓝 藻共生体 $\left(0.85 \mathrm{~kg} \cdot \mathrm{hm}^{-2} \cdot \mathrm{a}^{-1}\right)$ 和泥炭藓-蓝藻共生体 $\left(0.81 \mathrm{~kg} \cdot \mathrm{hm}^{-2} \cdot \mathrm{a}^{-1}\right)$ 的固氮量平均值高于塔藓-蓝藻共 生体 $\left(0.64 \mathrm{~kg} \cdot \mathrm{hm}^{-2} \cdot \mathrm{a}^{-1}\right)$, 混合苔藓-蓝藻共生体的固 氮量最低 $\left(0.53 \mathrm{~kg} \cdot \mathrm{hm}^{-2} \cdot \mathrm{a}^{-1}\right)($ 图2)。森林中的苔藓植 物种类繁多, 研究森林中的不同种类苔藓-蓝藻共 生体的固氮能力, 能更准确地评估森林中由苔藓蓝藻固定的氮的输入量, 可为森林中的氮循环研究 提供更准确的科学依据。

\section{3 苔藓-蓝藻共生体所固定氮的去向}

有研究发现, 藓-藻共生体所固定的氮只有 $20 \%$ 留存于藻类, 其他的转移至苔藓植物内(Meeks et al., 2002; Adams \& Duggan, 2012; Rousk et al., 2016f)。转移至苔藓植物体内的氮能通过菌根真菌 送至其他高等植物的根部被利用(Carleton \& Read, 


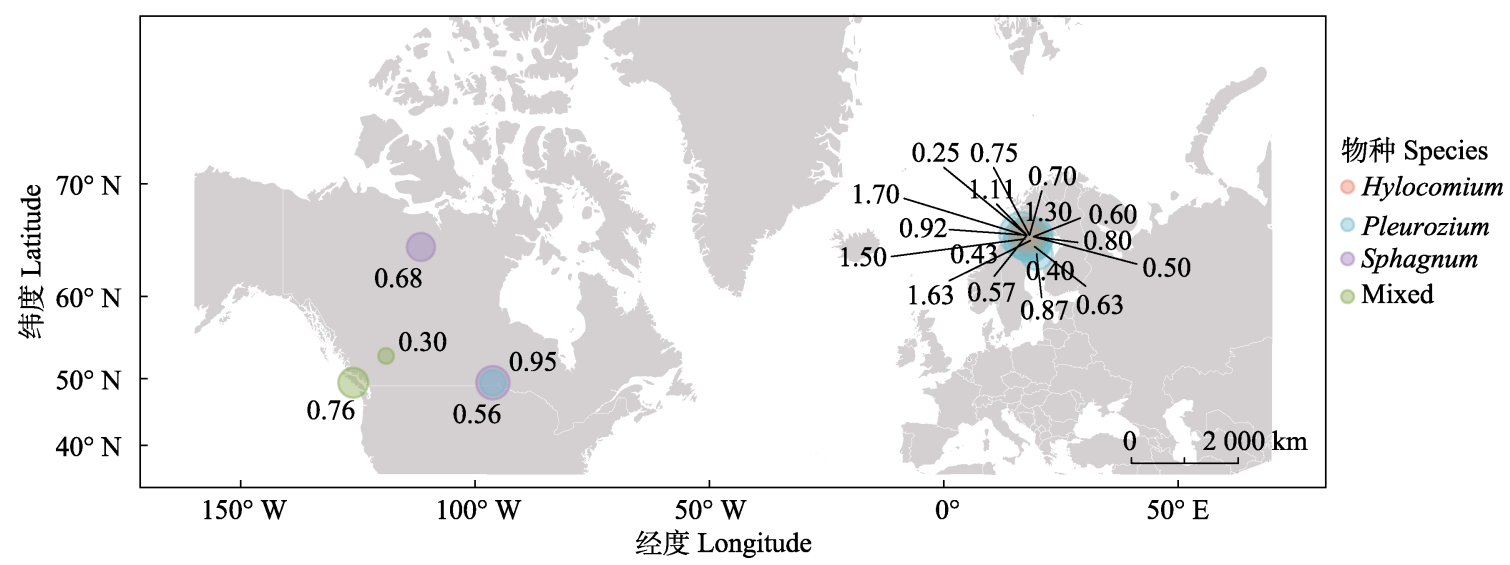

图2 不同区域苔藓-蓝藻共生体固氮能力 $\left(\mathrm{kg} \cdot \mathrm{hm}^{-2} \cdot \mathrm{a}^{-1}\right)$ 。图中数据来源于: Blundon \& Dale, 1990; Deluca et al., 2002b; Lagerstorm et al., 2007; Markham, 2009; Zackrisson et al., 2009; Gundale et al., 2011; Lindo \& Whiteley, 2011; Stewart et al., 2011a; Rousk et al., 2013b; Stuiver et al., 2015。

Fig. 2 Nitrogen fixation capacities $\left(\mathrm{kg} \cdot \mathrm{hm}^{-2} \cdot \mathrm{a}^{-1}\right)$ of bryophyte-cyanobacteria symbioses in different sites. All the data in the figure are from: Blundon \& Dale, 1990; Deluca et al., 2002b; Lagerstorm et al., 2007; Markham, 2009; Zackrisson et al., 2009; Gundale et al., 2011; Lindo \& Whiteley, 2011; Stewart et al., 2011a; Rousk et al., 2013b; Stuiver et al., 2015.

2011)。目前对苔藓植物体内的氮转移方式除菌根真 菌方式外, 还有受到干扰(如火烧)后氮释放至土壤 中, 但速率很慢(Rousk et al., 2013c)。由于多数苔藓 与蓝藻之间以附生的形式共生，两者之间没有特化 的结构用于转移固定的氮, 推测蓝藻固定的氮未被 宿主苔藓吸收而直接释放到环境中的可能性亦存在, 特别是在降水频繁的季节(Coxson, 1991; Wilson \& Coxson, 1999)。然而, 目前针对泥炭藓属少数物种 的研究认为大部分共生体固定的氮最终都转移到了 苔藓体内(Rai et al., 2002; Rousk et al., 2016f)。总体 而言, 定量地研究苔藓-蓝藻共生体所固定氮的去向 的报道很少, 共生体所固定氮的去向与氮转移过程 及其机理仍然很不清楚。

转移至苔藓植物体内的氮以及苔藓自身的氮可 能通过以下 3 种途径进一步为其他植物所利用。(1) 苔藓植物体中的养分将逐渐通过调落物分解过程而 释放(Turetsky et al., 2008)。苔藓凋落物的分解速率 低于其他高等植物调落物(杨林等, 2015), 因此通过 苔藓调落物分解释放氮将是速度慢且周期长的过 程。(2)苔藓体内部分养分和碳水化合物将在干湿交 替和冻融交替的过程中淋失(Coxson, 1991; Wilson \& Coxson, 1999)。但迄今为止, 苔藓氮淋失占苔藓 体内氮的比例, 以及氮淋失在生态系统氮循环中的 作用仍然有待进一步量化的研究。(3)苔藓体内的氮 通过菌根真菌被邻近其他植物利用。放射性同位素 标记实验表明, 菌根真菌将磷和氮转运到欧洲赤松 (Pinus sylvestris)的根系中(Carleton \& Read, 1991)。
虽然这一过程也可能是经过苔藓的养分淋失实现的, 但有研究证实, 苔藓植株确实能够受菌根真菌侵染 (Zhang \& Guo, 2007)。

\section{4 影响苔藓-蓝藻共生体固氮的因素及其作 用机理}

\section{1 水分、温度、光照及其综合影响}

苔藓与蓝藻均是变水植物, 对环境中的水分变 化十分敏感, 各自均在失水-复水过程中形成了独 特的变水适应机制。因此, 水分对苔藓-蓝藻共生体 关系及其固氮能力的影响很大。水分增加能使苔藓蓝藻共生体的固氮能力加强(Gundale et al., 2009, 2012b; Jackson et al., 2011)。因为苔蘚是敏感的变水 植物，水分含量变化能显著改变其生理生化过程 (李佑稷等, 2004; Rousk et al., 2013d), 因而可能会 导致依存苔藓的蓝藻的生长发生改变。已经发现, 连续干燥 3 天后苔藓-蓝藻共生体失去固氮能力(小 于 $0.2 \mu \mathrm{mol} \cdot \mathrm{m}^{-2} \cdot \mathrm{h}^{-1}$ ), 虽在复水过程中能缓慢恢复, 但需 5 天左右才能恢复原有固氮水平(Proctor, 2001; Rousk et al., 2013d), 可见水对蓝藻的固氮能力影响 很大, 所以水是苔藓-蓝藻共生体固氮过程中的关 键因子(Gundale et al., 2012b)。

两种大镰刀藓(Drepanocladus exannulatus、D. trichophyllus) 和岸生泥炭藓的固氮共生体在 11$16{ }^{\circ} \mathrm{C}$ 时具有最大的固氮能力(Granhall \& Selander, 1973; Gentili et al., 2005 ); 赤茎藓和塔藓与蓝藻的 共生体均在温度高于 $30.3{ }^{\circ} \mathrm{C}$ 时固氮速率明显下降, 
但比较而言赤茎藓-蓝藻共生体比塔藓-蓝藻共生体 对温度的耐受强度更高(Gundale et al., 2012a)。北方 森林中年平均温度的升高会使苔藓-蓝藻共生体的 固氮率增加(Houlton et al., 2008), 然而过高的温度 $\left(32{ }^{\circ} \mathrm{C}\right)$ 会影响苔藓-蓝藻共生体的氮固定速率 (Zielke et al., 2002); 将分离出的与苔藓共生的念珠 藻(NostoC sp.)和眉藻(Calothrix sp.)在不同温度下的 固氮活性比较发现, 两者的固氮速率迥异。在低温 $\left(5{ }^{\circ} \mathrm{C}\right)$ 下, 念珠藻具有低的固氮活性, 而眉藻在此 温度下无固氮能力; 在高温 $\left(30{ }^{\circ} \mathrm{C}\right)$ 下, 眉藻却具有 最高的固氮活性, 几乎是同等温度下念珠藻固氮活 性的3倍(Gentili et al., 2005), 蓝藻的不同最适固氮 温度可能是导致苔藓-蓝藻共生体固氮作用季节差 异的原因(Deluca et al., 2002b; Zackrisson et al., 2004)。此外, 温度升高亦使得固氮酶的启动推迟, 使蓝藻的固氮速率降低(Gundale et al., 2009; Stewart et al., 2011b)。在水分不受限的情况下, 0-10 ${ }^{\circ} \mathrm{C}$ 时共生体固氮率较低; 高于 $10{ }^{\circ} \mathrm{C}$ 时温度升高会使 固氮速率快速提高(Vitousek et al., 2002; 苏延桂等, 2011; Rousk et al., 2017b), 因为固氮酶能耐受的最 高温在 $25{ }^{\circ} \mathrm{C}$ 左右, 所以固氮最高温在 $25{ }^{\circ} \mathrm{C}$ 左右。 Zielke等(2002)发现自由生长的蓝藻固氮最高温为 $25{ }^{\circ} \mathrm{C}$, 但和苔藓共生后的共生体其固氮最高温为 $32{ }^{\circ} \mathrm{C}$ 。因此, 温度对蓝藻固氮能力有较大影响, 而 与苔藓形成共生关系更有利于氮固定, 可能是因为 与苔藓共生后, 改变了蓝藻固氮适宜温度范围。

Rousk等(2017b)对赤茎藓和毛青藓的蓝藻共生体进 行水分 $(0 、 25 \% 、 100 \%)$ 和温度 $\left(10 、 20 、 30{ }^{\circ} \mathrm{C}\right)$ 双因 素对共生体固氮能力的影响研究, 发现毛青藓-蓝 藻共生体固氮速率受水分含量的影响较大, 且两种 共生体在高的水分含量下具有更大的固氮酶活性, 固氮最适温度在 $20-30{ }^{\circ} \mathrm{C}$ ，与苏延桂等(2011)对荒 漠土壤生物结皮固氮酶活性的研究及Vitousek等 (2002)的结果类似。

蓝藻固氮作用的过程对光具有依赖性。光可以 通过光合作用影响其固氮过程中的电子供应链, 或 是影响固氮必需的高能量物质的产生。蓝藻固氮酶 的活性也受到光的调节, 不同的光照强度下固氮活 性呈现相应的高低差异, 且光照可缓解因添加结合 态氮对固氮作用的抑制效应(陈因和方大惟, 1983)。 自由生长的蓝藻的固氮作用不能离开光照, 与苔藓 附生的藓-蓝藻共生体的固氮作用同样受到光的较
大影响。Basilier等(1978)发现泥炭藓(Sphagnum sp.) 和镰刀藓-蓝藻共生体的固氮活性受到光的强烈影 响, 但内生于苔藓体内的蓝藻是否可以通过苔藓的 光合系统供应其固氮所需的电子, 从而使其对光的 依赖性降低，尚未见相关报道。

在最适温度下, 提高光照强度会增加共生体固 氮能力。Gentili等(2005)发现, 同在 $13{ }^{\circ} \mathrm{C}$ 时, 光照强 度 $160 \mu \mathrm{mol} \cdot \mathrm{m}^{-2} \cdot \mathrm{s}^{-1}$ 下的共生体固氮能力大于光照 强度为 $80 \mu \mathrm{mol} \cdot \mathrm{m}^{-2} \cdot \mathrm{s}^{-1}$ 时的固氮能力。目前已发现 赤茎藓-蓝藻共生体的最适温度-光照综合值为 $23{ }^{\circ} \mathrm{C} 、 130 \mu \mathrm{mol} \cdot \mathrm{m}^{-2} \cdot \mathrm{s}^{-1}$ (Gentili et al., 2005)和 $22^{\circ} \mathrm{C}$ 、 $500 \mu \mathrm{mol} \cdot \mathrm{m}^{-2} \cdot \mathrm{s}^{-1}$, 而塔藓-蓝藻共生体最适温度-光 照综合值为 $16.3{ }^{\circ} \mathrm{C} 、 500 \mu \mathrm{mol} \cdot \mathrm{m}^{-2} \cdot \mathrm{s}^{-1}$ (Gundale et al., 2012a)。两研究中赤茎藓的最适温度-光照值中温度 值相似, 但是光照值迥异, Gentili等(2005)未设置23 ${ }^{\circ} \mathrm{C}$ 下更高的光照强度, 未能明确更高光照是否增加 其固氮能力, 这可能是两研究结果差异的原因。明 确温度和光照等非生物因素的综合作用对生物固氮 的影响, 可以预测和模拟森林环境中氮的循环, 及 森林冠层下的苔藓层氮输入在气候变化中引起的潜 在反馈效应(Sorensen \& Michelsen, 2011), 但是目前 对非生物因子作用于苔藓-蓝藻共生体固氮能力的 综合影响的研究十分贫乏。

\section{2 氮、磷及金属元素的影响}

苔藓为蓝藻提供了适宜的生存环境, 苔藓的生 物量决定的苔藓种群结构直接影响到蓝藻的生存环 境, 进而影响到苔藓-蓝藻共生体的固氮作用。大气 沉降的养分是苔藓养分的主要来源, 因此养分对苔 藓-蓝藻共生体固氮作用的影响值得特别关注。

目前有 4 个实验揭示了大气氮沉降对藓-蓝藻共 生体固氮能力的影响。氮沉降量的增加能减少苔藓 生物量(Gundale et al., 2013; Du et al., 2014), 共生 体的固氮能力相应下降(Gundale et al., 2013; Stuiver et al., 2015)。另外, 苔藓对氮沉降的敏感程度因物 种而异(Rousk \& Michelsen, 2016)。长期实验发现, 塔藓-蓝藻共生体的生物量和固氮能力受氮沉降影 响的程度高于赤茎藓-蓝藻共生体(Gundale et al., 2013)。苔藓-蓝藻共生体对氮沉降的响应存在明显 的剂量效应 $($ dose-effect) 关系。在氮沉降量较低 $(<3$ $\left.\mathrm{kg} \cdot \mathrm{hm}^{-2}\right)$ 时, 苔藓-蓝藻共生体的固氮能力与对照 (无额外氮沉降)无明显差异(Zackrisson et al., 2009; Gundale et al., 2011); 氮沉降量在3-12 kg. hm ${ }^{-2}$ 之间 
时, 苔藓-蓝藻共生体的固氮速率出现不同程度的 下降, 苔藓植物体表面附生的蓝藻数量也呈现相应 的下降趋势(Gundale et al., 2011; Rousk et al., 2013e); 氮沉降在较高水平 $\left(>12 \mathrm{~kg} \cdot \mathrm{hm}^{-2}\right)$ 下, 固氮作用停滞, 苔藓植物体表面几乎没有蓝藻附生(Jones et al., 2004; Gundale et al., 2011; Rousk et al., 2013a, $2013 \mathrm{e})$ 。也有研究发现氮添加量为 $10 \mathrm{~kg} \cdot \mathrm{hm}^{-2} \cdot \mathrm{a}^{-1}$ 时, 赤茎藓共生体的固氮作用并没有受到限制(Rousk, 2013a), 但当氮沉降大于 $10 \mathrm{~kg} \cdot \mathrm{hm}^{-2} \cdot \mathrm{a}^{-1}$ 时, 其固氮 作用受到显著抑制(Rousk et al., 2013b)。表明苔藓蓝藻共生体的固氮速率确实受到氮沉降量的影响, 但具体阈值以及阈值的种间差异仍然有待进一步 研究。

苔藓-蓝藻共生体对磷的响应, 或者说磷对苔 藓-蓝藻共生体固氮能力的影响, 目前还难有确切 定论, 相关结果仍然是矛盾的。Chapin等(1991)发现, 添加磷时北极柳(Salix arctica)环境下的苔藓体固氮 能力均增加, 塔藓和赤茎藓-蓝藻固氮能力亦增加 (Rousk et al., 2017a); 但Zackrisson等(2004, 2009)对 赤茎藓和塔藓-蓝藻共生体的磷添加 $\left(5 \mathrm{~kg} \cdot \mathrm{hm}^{-2} \cdot \mathrm{a}^{-1}\right)$ 实验表明, 磷的添加对其固氮能力并无影响; Smith (1984)将Brachythecium subplicatum-蓝藻共生体置 于磷浓度为 $2.5 \mu \mathrm{g} \cdot \mathrm{mL}^{-1}$ 的液体中培养, 发现磷的添 加抑制其固氮能力, 此种培养方式与土壤培养方式 的不同, 可能是导致结果差异的原因, 且磷添加对 共生体固氮作用亦可能存在种间差异及阈值, 但目 前尚未见明确报道。上述养分冲突性结果表明, 未 来需要更多且更深入的苔藓-蓝藻共生体对磷的响 应的研究。此外, 最近Rousk等(2017a)发现, 添加重 金属钼(Mo)增加了塔藓-蓝藻共生体和赤茎藓-蓝藻 共生体的固氮能力, 且单独添加钼比单独添加磷和 磷及钼的混合添加能更好地提升共生体中的固氮酶 活性, 他们指出苔藓-蓝藻共生体的固氮作用受到 重金属钼的限制, 且钼对蓝藻的生物量有影响。

\section{$4.3 \mathrm{pH}$ 值的影响}

已经发现环境 $\mathrm{pH}$ 值能显著影响藓-蓝藻共生关 系及其固氮能力。当 $\mathrm{pH}$ 值为 3.8 时, 3 种泥炭藓(Sphagnum balticum、锈色泥炭藓(S. fuscum)和垂枝泥炭 藓(S. jensenii)) 完全没有藻类与之共生; 在 $\mathrm{pH}$ 值为 4.2和4.9的环境中, S. lindebergii和岸生泥炭藓有藻 类内生; 在 $\mathrm{pH}$ 值更高的环境中, 藻类附生或自由生 长(Granhall \& Selander, 1973); 蓝藻在自由生长时
的最适环境 $\mathrm{pH}$ 值为 $5-10$, 且在培养过程中酸性环境 $\mathrm{pH}$ 值上升, 而碱性环境 $\mathrm{pH}$ 值未发生较大的变化(罗 伟和沈健英, 2007; 郑云普等, 2010), 说明蓝藻自由 生长时更适宜碱性环境, 但是和泥炭藓共生后, 能 在更低pH值的环境中生存且固氮。Smith (1984)亦 发现Brachythecium subplicatum-藻类共生体于水体 培养时最适固氮 $\mathrm{pH}$ 值为5.9-6.2。由此, 蓝藻和泥炭 藓共生后能适应更严酷的环境条件。上述研究结果 来自于室内培养实验与野外调查, 但野外自然条件 下的实验研究贫乏, $\mathrm{pH}$ 对苔藓-蓝藻共生体的形成 以及固氮能力的影响程度及其机理还不清楚。

\section{4 森林垂直冠层和演替阶段的影响}

苔藓群落组成与生物量在森林的空间差异及其 随森林发育演替的变化是明显的, 必然引起苔藓一 蓝藻共生体关系及其固氮能力的变化。明确这样的 时空变化特点与规律十分必要。Lindo和Whiteley (2011)发现, 在海岸温带雨林中, 地表层为生态系 统提供的有效氮较冠层附生苔藓-蓝藻共生体少。从 该森林中 $30 、 15 \mathrm{~m}$ 冠层与地表的苔藓-蓝藻共生体固 氮量和总的蓝藻(伪枝藻属)细胞数目的比较都显示, 冠层的藻类数目最多, 固氮量最高, 而地表的氮固 定量最低(Lindo \& Whiteley, 2011)。受水分、风速和 光强的影响, 森林冠层的苔藓植物种类和生物量 都较亚冠层的苔藓植物种类少, 而氮固定量低 (Newmaster et al., 2003; Han et al., 2010)。

苔藓-蓝藻共生体的固氮能力在森林演替的后 期更高(Zackrisson et al., 2004; Stuiver et al., 2015), 原因比较复杂。目前认识到的原因有 4 个: 一是演替 后期虽然整个系统的氮量很大, 但可被苔藓直接吸 收的可利用氮量却很少(DeLuca et al., 2002a)。二 是幼林中地表苔藓层覆盖率比老林的少 $1 / 3$ 以上, 老林不管是冠层还是地表都具有对苔藓-蓝藻共生 体生长更有利的环境条件(Newmaster et al., 2003; Lagerstrom et al., 2007; Han et al., 2010)。三是因为 演替早期受到苔藓植物和蓝藻数量的限制, 其固氮 能力低(DeLuca et al., 2007); 也有人发现, 林木生 物量的增加和林下透光率的减少使苔藓-蓝藻共生 体(塔藓-蓝藻)的固氮能力下降(Stuiver et al., 2015)。 但是Arróniz-Crespo等(2014)对智利西南部达尔文山 脉(Tierra del Fuego)冰消区域植被不同发育时期(即 演替过程中)苔藓-蓝藻共生体固氮能力的研究显示, 在较为湿润的冰川消退早期(4-7年, 植被主要为石 
生苔藓植物), 共生体固氮能力最强, 为1.656-1.780 $\mathrm{kg} \cdot \mathrm{kg}^{-1} \cdot \mathrm{a}^{-1}$; 但是在较干燥的冰川消退中期(26-66 年, 植被主要为 $10 \mathrm{~m}$ 左右高的灌从或乔木), 苔藓蓝藻共生体的固氮能力次之, 为 0.338-0.511 $\mathrm{kg} \cdot \mathrm{kg}^{-1} \cdot \mathrm{a}^{-1}$; 晚期(80年)时, 其固氮能力最低, 仅为 $0.288 \mathrm{~kg} \cdot \mathrm{kg}^{-1} \cdot \mathrm{a}^{-1}$ 。上述结果表明, 森林垂直层次或 演替阶段对苔藓-蓝藻共生体的形成及其固氮能力 影响的差异性取决于微环境条件以及苔藓-蓝藻共 生体相互作用的强度与过程, 机理相当复杂。

\section{5 生物因素影响}

苔藓-蓝藻共生体关系显然受到形成共生体的 苔藓与蓝藻种类的影响, 可以推测也受其他可能影 响其微生境与关系的生物类群特性如乔木层类群、 灌草类群的影响。已发现, 由于蓝藻自身的固氮能 力差异及其对环境适应能力不同, 苔藓-蓝藻共生 体的固氮能力有差异(Zielke et al., 2009)。念珠藻是 目前所知藻类中能与多种苔藓共生固氮的种类, 能 与苔藓形成共生体的其他藻类数量较少, 而共生体 固氮能力因藻类不同种类的差异性及其机理尚不清 楚。苔藓能通过促使藻类异形胞数量的增加而增加 共生体的固氮率, 也能产生一种毒素物质抑制藻类 生长(Rousk, 2013a), 不同苔藓促进藻类异形胞数量 的能力不可能一样。因此, 共生苔藓种类也影响与 之共生的藻类种类及其固氮能力 (前面已有论述)。

Sanna等(2015)发现共生体固氮率大小取决于苔藓 种类, 泥炭藓-藻类共生体较森林中的其他藓-藻共 生体固氮能力强。目前尚缺乏严谨的实验去深入揭 示苔藓与藻类共生体的关系及其可能的固氮能力如 何受苔藓与藻类的影响, 更缺乏其他生物因素对苔 藓-蓝藻共生体关系及其功能影响的研究。

\section{5 结论和展望}

\section{1 结论和认识}

从过去 50 余年来苔藓-蓝藻共生体及其可能的 固氮能力研究可以归纳出一些初步结论和认识。

(1) 目前已知能形成苔藓-蓝藻共生体的苔藓有 35 属 41 种 (表 1$)$, 蓝藻有 8 属, 占全球已知苔藓和蓝 藻种类的比例很小。即使如此, 其中的大部分仍然 未能准确识别到物种水平。因此, 苔藓-蓝藻共生体 可能是广泛现象, 因为大多数已知藓种(如塔藓、赤 茎藓) 是广泛分布的种类, 但就苔藓与蓝藻的物种 多样性而言, 苔藓-蓝藻共生体的形成是否是普遍
的还不清楚。

(2)苔藓-蓝藻共生体可根据苔藓类群差异简单 分为 3 个类群: 角苔-蓝藻共生体、苔-蓝藻共生体和 藓-蓝藻共生体, 存在内生和附生两种共生关系; 角 苔与苔类和蓝藻的共生关系主要以内生为主, 而藓 类与蓝藻的共生关系以附生为主; 共生关系中苔藓 为蓝藻不仅提供了更稳定的微生境, 也有可能提供 营养物质(如碳水化合物)以启动或维持藻类定居与 生长, 从而改善蓝藻的固氮能力。

(3)藓-蓝藻共生体具有固氮能力, 而且比蓝藻 单独生长时的固氮能力更强, 但并不是所有藓-蓝 藻共生体均具有固氮能力; 角苔-蓝藻与苔-蓝藻共 生体固氮能力的强弱目前仍未有确切报道; 内生与 附生共生体的固氮能力差异性也不清楚。苔藓-蓝 藻共生固氮能力研究目前仅聚焦于藓-蓝藻类型, 而缺乏角苔-蓝藻与苔-蓝藻的共生体固氮能力的 报道。

(4)仍然有限的藓-蓝藻共生体的固氮能力及其 变化研究主要聚焦于北欧与北美高纬度北方针叶林 或北极圈附近地区的植被，其他地区(包括中国)的 案例研究非常贫乏。已报道的藓-蓝藻共生体固氮能 力为 $0.25-1.7 \mathrm{~kg} \cdot \mathrm{hm}^{-2} \cdot \mathrm{a}^{-1}$, 因区域差异与苔藓种类 不同而差异较大, 固氮能力变化研究的工作十分 缺乏。

(5)苔藓-蓝藻共生体关系及其固氮能力受共生 的藓与蓝藻的种类, 光、温、水等环境因素以及氮、 磷和钼等元素的影响, 但作用机制尚不清楚。

\section{2 需要聚焦的研究方向与科学问题}

苔藓-蓝藻共生体及其互作关系、固氮能力、影 响因素及其作用机理的认识仍然是粗浅的, 很多关 键问题仍不清楚, 需要更多更深入的研究去加深对 苔藓蓝藻共生体关系、效应与机制的科学认识, 深 化或突破我们对生物多样性维持机制与养分循环机 制的已有认识。现有工作和认识明显不足, 需要聚 焦。

\subsection{1 苔藓-蓝藻共生关系、动态变化及其互作机制}

苔藓与蓝藻间存在共生关系是明确的, 但如何 形成的? 苔藓释放的何种特定物质如何促进共生体 的形成? 共生关系是否在苔藓多样性中是普遍而广 泛的? 多数附生型苔藓-蓝藻共生体并没有特化的 附生结构, 但与藓类附生却大大加强了蓝藻固氮能 力, 这种附生形式的共生体是否广泛存在于苔藓物 
种与固氮蓝藻之间? 这些基础性问题并不清楚。因 此, 加强苔藓-蓝藻共生关系、动态变化及其互作机 制的研究是必要的, 不仅有助于强化对苔藓-蓝藻 共生体形成、演变与机理的规律性与一般性的深入 认识, 也能形成和推动跨植物类群多样性维持机制 的创新性认识。现有苔藓-蓝藻共生体关系的研究主 要是采用形态学手段进行初步的静态观察, 藓-藻 共生体关系的定量的分子生物学研究证据缺乏必要 的应用。采取严谨的生态学实验方法, 开展苔藓-蓝 藻共生关系及其互作机制的分子生物学基础研究是 当前亟待展开的重要突破方向。

\subsection{2 苔藓-蓝藻共生体的固氮效应及其动态变化 与驱动机制}

对苔藓-蓝藻共生体的固氮效应及其动态变化 的认识严重不足。苔藓如何调控共生体的固氮作 用? 内生固氮和附生固氮的固氮效率是否有明显的 差异? 共生体关系及其固氮效应如何变化? 互动关 系如何相互驱动? 共生体所固定的氮如何转移? 转 移途径、方式及其数量怎样变化? 这些基础性理论 问题大大制约着苔藓-蓝藻共生体固氮效应与机理 研究的深入。

\subsection{3 苔藓-蓝藻共生体固氮能力与生态环境变化 的关系与作用机理}

苔藓-蓝藻共生体关系及其固氮能力对环境(气 候)变化十分敏感, 逐渐成为全球气候变化研究的 重要对象(Gundale et al., 2012a)。在北欧所开展的几 个生态学实验包括大气 $\mathrm{N}$ 沉降、增温以及降水变化 的模拟实验研究, 获得了一些新的事实依据, 但大 多仅针对少数单个因子的效应认识, 缺乏对多环境 因子综合作用效应的案例研究。全球气候变化的区 域差异性相当明显, 因此, 从气候变化角度获得的 那些基本认识在全球范围内的普遍性以及区域差异 性仍然需要更多的研究进一步证实和完善, 以加深 对气候变化程度与苔藓-蓝藻共生体固氮能力的定 量关系的理论认识。森林对全球气候变化具有很强 的调节作用, 全球变化通过森林调控而作用于林下 苔藓-蓝藻共生体, 因此, 森林环境变化与全球气候 变化至少在程度上是有些差异的, 揭示苔藓-蓝藻 共生体固氮能力与林下生态环境变化的关系十分必 要。更重要的是, 当前环境变化与苔藓-蓝藻共生体 固氮能力的关系在生理学、生物学与生态及其化学 方面的机理认识十分贫乏, 应该成为一个重要研究
方向。在苔藓-蓝藻共生体固氮的关键区域(例如高 山和亚高山森林生态系统中), 结合多种森林环境 因子监控, 进行长期野外观测, 深入研究环境变化 对苔藓-蓝藻共生体固氮的影响, 将有助于评估共 生体固氮对该生态系统的实际贡献, 并可模拟和预 测未来环境变化对生态系统氮循环的影响。

\subsection{4 苔藓-蓝藻共生体固氮能力时空格局变化规 律及其在森林生态系统氮循环中的贡献}

到目前为止, 有限的研究为认识苔藓-蓝藻共 生体固氮能力在生态系统氮输入中的重要性提供了 明确的证据和新的视角, 无疑为生态系统氮循环过 程及其机制的认识带来了新的希望。要充分认识贫 㾉生态系统演替中养分驱动机制, 急需开展更多更 广泛的苔藓-蓝藻共生体固氮研究, 以明确不同区 域生态系统苔藓-蓝藻共生体固氮能力的时空格局 变化规律, 厘清苔藓-蓝藻共生体固氮在森林生态 系统氮循环中的贡献率。在自然条件下的实验生态 学方法与长期定位研究方法的有机结合是这方面研 究在方法上的必然选择, 亟待强化。此外, 鉴于苔藓 在高山、亚高山生态系统, 北方针叶林以及北极圈 生态系统中的优势程度, 这些区域苔藓-蓝藻共生 体固氮能力的研究应该是重点关注的问题。在区域 生态系统养分循环模型研究与应用中应充分考虑苔 藓以及苔藓-蓝藻共生体在氮循环中的作用。强化苔 藓-蓝藻共生体关系及其形成机制、固氮过程与环境 变化的关系及其作用机理的研究将有助于准确预测 森林的动态, 以及更充分地认识苔藓在森林生态系 统氮循环中的作用。

致谢 感谢中国科学院成都生物研究所问晓丽、庞 学勇、李芳兰、胡斌、杨兵和冯德枫等在论文写作 过程中所提供的帮助与修改完善建议。

\section{参考文献}

Adams DG, Duggan PS (2008). Cyanobacteria-bryophyte symbioses. Journal of Experimental Botany, 59, 1047- 1058.

Adams DG, Duggan PS (2012). Signalling in CyanobacteriaPlant Symbioses. Signaling and Communication in Plant Symbiosis. Springer, Berlin. 11, 93-121.

Arróniz-Crespo M, Pérez-Ortega S, De los Ríos A, Green TGA, Ochoa-Hueso R, Casermeiro MÁ, de la Cruz MT, Pintado A, Palacios D, Rozzi R, Tysklind N, Sancho LG (2014). Bryophyte-cyanobacteria associations during primary succession in recently deglaciated areas of Tierra del Fuego (Chile). PLOS ONE, 9, e96081. DOI: 10.1371/journal. 
pone. 0096081 .

Basilier K, Granhall U, Stenström TA (1978). Nitrogen fixation in wet minerotrophic moss communities of a subarctic mire. Oikos, 31, 236-246.

Bay G, Nahar N, Oubre M, Whitehouse MJ, Wardle DA, Zackrisson O, Nilsson MC, Rasmussen U (2013). Boreal feather mosses secrete chemical signals to gain nitrogen. New Phytology, 200, 54-60.

Berg A, Danielsson Å, Svensson BH (2012). Transfer of fixed-N from $\mathrm{N}_{2}$-fixing cyanobacteria associated with the moss Sphagnum riparium results in enhanced growth of the moss. Plant and Soil, 362, 271-278.

Blundon DJ, Dale MRT (1990). Dinitrogen fixation (acetylene reduction) in primary succession near Mount Robson, British Columbia, Canada. Arctic Antarctic and Alpine Research, 22, 255-263.

Carleton TJ, Read DJ (1991). Ectomycorrhizas and nutrient transfer in conifer-feather moss ecosystems. Canadian Journal of Botany, 69, 778-785.

Chapin DM, Bliss LC, Bledsoe LJ (1991). Environmentregulation of nitrogen-fixation in a high arctic lowland ecosystem. Canadian Journal of Botany, 69, 2744-2755.

Chen Y, Fang DW (1983). Light-regulation of nitrogen fixation in the blue-green algae Anabaena 7120. Acta Phytophysiologia Sinica, 9, 51-59. [陈因, 方大惟 (1983). 蓝藻 Anabaena 7120固氮的光调节. 植物生理学报, 9, 51-59.]

Cleveland CC, Townsend AR, Schimel DS, Fisher H, Howarth RW, Hedin LO, Perakis SS, Latty EF, Von Fischer JC, Elseroad A, Wasson MF (1999). Global patterns of terrestrial biological nitrogen $\left(\mathrm{N}_{2}\right)$ fixation in natural ecosystems. Global Biogeochemical Cycles, 13, 623-645.

Cornelissen JHC, Lang SI, Soudzilovskaia NA, During HJ (2007). Comparative cryptogam ecology: A review of bryophyte and lichen traits that drive biogeochemistry. Annals of Botany, 99, 987-1001.

Coxson DS (1991). Nutrient release from epiphytic bryophytes in tropical montane rain forest (Guadeloupe). Canadian Journal of Botany, 69, 2122-2129.

Davey A, Marchant HJ (1983). Seasonal variation in nitrogen fixation by Nostoc commune Vaucher at the Vestfold Hills, Antarctica. Phycologia, 22, 377-385.

Deluca T, Zackrisson O, Gundale M, Nilsson MC (2008). Ecosystem feedbacks and nitrogen fixation in boreal forests. Science, 320, 1181.

Deluca TH, Nilsson MC, Zackrisson O (2002a). Nitrogen mineralization and phenol accumulation along a fire chronosequence in northern Sweden. Oecologia, 133, 206-214.

Deluca TH, Zackrisson O, Gentili F, Sellstedt A, Nilsson MC (2007). Ecosystem controls on nitrogen fixation in boreal feather moss communities. Oecologia, 152, 121-130.

Deluca TH, Zackrisson O, Nilsson MC, Sellstedt A (2002b). Quantifying nitrogen-fixation in feather moss carpets of boreal forest. Nature, 419, 917-920.

Du EZ, Liu XY, Fang JY (2014). Effects of nitrogen additions on biomass, stoichiometry and nutrient pools of moss Rhytidium rugosum in a boreal forest in northeast China. Environmental Pollution, 188, 166-171.

Gavazov KS, Soudzilovskaia NA, van Logtestijn RSP, Braster M, Cornelissen JHC (2010). Isotopic analysis of cyanobacterial nitrogen fixation associated with subarctic lichen and bryophyte species. Plant and Soil, 333, 507-517.

Gentili F, Nilsson MC, Zackrisson O, Deluca TH, Sellstedt A (2005). Physiological and molecular diversity of feather moss associative $\mathrm{N}_{2}$-fixing cyanobacteria. Journal of $E x$ perimental Botany, 56, 3121-3127.

Gerber S, Hedin LO, Oppenheimer M, Pacala SW, Shevliakova E (2010). Nitrogen cycling and feedbacks in a global dynamic land model. Global Biogeochemical Cycles, 24, 723-738.

Granhall U, Basilier K (1973). Nitrogen fixation in tundra moss communities. Progress Report, Swedish IBP. Tundra Biome Project Technical Report, 14, 174-190.

Granhall U, Selander H (1973). Nitrogen fixation in a subarctic mire. Oikos, 24, 8-15.

Gundale MJ, Bach LH, Nordin A (2013). The impact of simulated chronic nitrogen deposition on the biomass and $\mathrm{N}_{2}$-fixation activity of two boreal feather moss-cyanobacteria associations. Biology Letters, 9, 1-4.

Gundale MJ, Deluca TH, Nordin A (2011). Bryophytes attenuate anthropogenic nitrogen inputs in boreal forests. Global Change Biology, 17, 2743-2753.

Gundale MJ, Gustafsson H, Nilsson MC (2009). The sensitivity of nitrogen fixation by a feathermoss-cyanobacteria association to litter and moisture variability in young and old boreal forests. Canadian Journal of Forest Research, 39, 2542-2549.

Gundale MJ, Nilsson M, Bansal S, Jaderlund A (2012a). The interactive effects of temperature and light on biological nitrogen fixation in boreal forests. New Phytologist, 194, 453-463.

Gundale MJ, Wardle DA, Nilsson MC (2012b). The effect of altered macroclimate on $\mathrm{N}$-fixation by boreal feather mosses. Biology Letters, 8, 805-808.

Han B, Zou XM, Kong JJ, Sha LQ, Gong HD, Yu Z, Cao T (2010). Nitrogen fixation of epiphytic plants enwrapping trees in Ailao Mountain cloud forests, Yunnan, China. Protoplasma, 247, 103-110.

Houle D, Bilodeau GS, Paquet S, Planas D, Warren A (2006). Identification of two genera of $\mathrm{N}_{2}$-fixing cyanobacteria growing on three feather moss species in boreal forests of Quebec, Canada. Canadian Journal of Botany, 84, 10251029.

Houlton BZ, Wang YP, Vitousek PM, Field CB (2008). A unifying framework for dinitrogen fixation in the terrestrial

www.plant-ecology.com 
biosphere. Nature, 454, 327-330.

Jackson BG, Martin P, Nilsson MC, Wardle DA (2011). Response of feather moss associated $\mathrm{N}_{2}$ fixation and litter decomposition to variations in simulated rainfall intensity and frequency. Oikos, 120, 570-581.

Jones MLM, Wallace HL, Norris D, Brittain SA, Haria S, Jones RE, Rhind PM, Reynolds BR, Emmett BA (2004). Changes in vegetation and soil characteristics in coastal sand dunes along a gradient of atmospheric nitrogen deposition. Plant Biology, 6, 598-605.

Lagerstrom A, Nilsson MC, Zackrisson O, Wardle DA (2007). Ecosystem input of nitrogen through biological fixation in feather mosses during ecosystem retrogression. Functional Ecology, 21, 1027-1033.

Li YJ, Li J, Chen J, Huang GW (2004). Responses of Dolichomitriopsis diveirsiformis photosynthesis rate to light, air temperature and plant water content. Chinese Journal of Applied Ecology, 15, 391-395. [李佑稷, 李菁, 陈军, 黄国文 (2004). 不同含水量下尖叶拟船叶藓光合速率 对光温的响应及其模型. 应用生态学报, 15, 391-395.]

Lindo Z, Whiteley JA (2011). Old trees contribute bio-available nitrogen through canopy bryophytes. Plant and Soil, 342, 141-148.

Liu JH, Bao WK, Li FL (2005). Major bryophyte patch structures and their relationships with environmental factors under a coniferous forest of eastern Tibetan plateau. Ecology and Environment, 14, 735-741. [刘俊华, 包维楷, 李 芳兰 (2005). 青藏高原东部原始林下地表主要苔藓斑 块特征及其影响因素. 生态环境学报, 14, 735-741.]

Liu X, Bao WK (2014). Understory plant assemblages present distinct short-term responses to the clear-cutting of an old-growth spruce forest near an alpine timberline. Canadian Journal of Forest Research, 44, 562-571.

Luo W, Shen JY (2007). Responses of growth of two Cyanobacteria species to different $\mathrm{pH}$ environments. Journal of Shanghai Jiaotong University (Agriculture Science), 25, 566-569. [罗伟, 沈健英 (2007). 2种蓝藻的生长对不同 环境 $\mathrm{pH}$ 的响应. 上海交通大学学报(农业科学版), 25, 566-569.]

Markham JH (2009). Variation in moss-associated nitrogen fixation in boreal forest stands. Oecologia, 161, 353-359.

Meeks JC (1990). Cyanobacterial-Bryophyte Associations. Chemical Rubber Company Press, Boca Raton.

Meeks JC (2007). Physiological adaptations in nitrogen-fixing Nostoc-plant symbiotic associations. In: Pawlowski K ed. Prokaryotic symbionts in plants. Microbiology Monographs. Vol. 8. Springer, Berlin.

Newmaster SG, Belland RJ, Arsenault A, Vitt DH (2003). Patterns of bryophyte diversity in humid coastal and inland cedar-hemlock forests of British Columbia. Environmental Reviews, 11, 159-185.

Proctor M (2001). Patterns of desiccation tolerance and reco- very in bryophytes. Plant Growth Regulation, 35, 147156.

Rai AN, Bergman B, Rasmussen U (2002). Cyanobacteria in Symbiosis. Springer, Berlin.

Rai AN, Trumbore SE, Davison EA, Harden JW, Veldhuis H (2000). Cyanobacterium-plant symbioses. New Phytologist, 174, 449-481.

Reddy GB, Giddens J (1981). Nitrogen fixation by moss-algal associations in grassland. Soil Biology and Biochemistry, 13, 537-538.

Reed SC, Cleveland CC, Townsend AR (2011). Functional ecology of free-living nitrogen fixation: A contemporary perspective. Annual Review of Ecology, Evolution, and Systematics, 42, 489-512.

Rippka R, Deruelles J, Waterbury JB, Herdman M, Stanier RY (1979). Generic assignments, strain histories and properties of pure cultures of cyanobacteria. Journal of General Microbiology, 11, 1-61.

Rosén K, Lindberg T (1980). Biological nitrogen fixation in coniferous forest watershed areas in central Sweden. Ecography, 3, 137-140.

Rousk K, Degboe J, Michelsen A, Bradley R, Bellenger JP (2017a). Molybdenum and phosphorus limitation of mossassociated nitrogen fixation in boreal ecosystems. New Phytologist, 214, 97-107.

Rousk K, Deluca TH, Rousk J (2013a). The cyanobacterial role in the resistance of feather mosses to decompositionToward a new hypothesis. PLOS ONE, 8, e62058. DOI: 10.1371/journal.pone.0062058.

Rousk K, Jones DL, Deluca TH (2013b). Exposure to nitrogen does not eliminate $\mathrm{N}_{2}$ fixation in the feather moss Pleurozium schreberi (Brid.) Mitt. Plant and Soil, 374, 513-521.

Rousk K, Jones DL, Deluca TH (2013c). Moss-cyanobacteria associations as biogenic sources of nitrogen in boreal forest ecosystems. Frontiers in Microbiology, 4, 150.

Rousk K, Jones DL, Deluca TH (2013d). The resilience of nitrogen fixation in feather moss (Pleurozium schreberi)cyanobacteria associations after a drying and rewetting cycle. Plant and Soil, 377, 159-167.

Rousk K, Michelsen A (2016). The sensitivity of mossassociated nitrogen fixation towards repeated nitrogen input. PLOS ONE, 11, e0146655. DOI: 10.1371/journal. pone. 0146655 .

Rousk K, Pedersen PA, Dyrnum K, Michelsen A (2017b). The interactive effects of temperature and moisture on nitrogen fixation in two temperate-arctic mosses. Theoretical and Experimental Plant Physiology, 29, 25-36.

Rousk K, Rousk J, Jones DL, Zackrisson O, Deluca TH (2013e). Feather moss nitrogen acquisition across natural fertility gradients in boreal forests. Soil Biology and Biochemistry, 61, 86-95.

Rousk K, Sorensen PL, Michelsen A (2016f). Nitrogen transfer 
from four nitrogen-fixer associations to plants and soils. Ecosystems, 19, 1491-1504.

Sanna ML, Antti JR, Marja T (2015). Nitrogen fixation in Sphagnum mosses is affected by moss species and water table level. Plant and Soil, 389, 185-196.

Smith VR (1984). Effects of abiotic factors on acetylene reduction by cyanobacteria epiphytic on moss at a subantarctic island. Applied and Environmental Microbiology, 48, 594600 .

Sorensen PL, Michelsen A (2011). Long-term warming and litter addition affects nitrogen fixation in a subarctic heath. Global Change Biology, 17, 528-537.

Stewart KJ, Coxson D, Grogan P (2011a). Nitrogen inputs by associative cyanobacteria across a low arctic tundra landscape. Arctic Antarctic and Alpine Research, 43, 267-278.

Stewart KJ, Lamb EG, Coxson DS, Siciliano SD (2011b). Bryophyte-cyanobacterial associations as a key factor in $\mathrm{N}_{2}$-fixation across the Canadian Arctic. Plant and Soil, 344, 335-346.

Stewart WDP (1966). Nitrogen Fixation in Plants. Athlone Press, London.

Stuiver BM, Gundale MJ, Wardle DA, Nilsson MC (2015). Nitrogen fixation rates associated with the feather mosses Pleurozium schreberi and Hylocomium splendens during forest stand development following clear-cutting. Forest Ecology and Management, 347, 130-139.

Su YG, Li XR, Zhao X, Li AX, Chen YW (2011). The nitrogenase activity of biological soil crusts and their responses to environmental factors. Advances in Earth Science, 26, 332-338. [苏延桂, 李新荣, 赵听, 李爱霞, 陈应武 (2011). 不同类型生物土壤结皮固氮活性及对环境因子 的响应研究. 地球科学进展, 26, 332-338.]

Tamm CO (1991). Nitrogen in Terrestrial Ecosystems. Springer, Berlin.

Turetsky MR, Crow SE, Evans RJ, Vitt DH, Wieder RK (2008). Trade-offs in resource allocation among moss species control decomposition in boreal peatlands. Journal of Ecology, 96, 1297-1305.

Vitousek PM, Cassman K, Cleveland C (2002). The Nitrogen Cycle at Regional to Global Scales. Springer, Berlin. $1-45$.

West NJ, Adams DG (1997). Phenotypic and genotypic com- parison of symbiotic and free-living cyanobacteria from a single field site. Applied and Environmental Microbiology, 63, 4479-4484.

Wilson JA, Coxson DS (1999). Carbon flux in a subalpine spruce-fir forest: Pulse release from Hylocomium splendens feather-moss mats. Canadian Journal of Botany, 77, 564-569.

Yang L, Deng CC, Chen YM, He RL, Zhang J, Liu Y (2015). Relationships between decomposition rate of leaf litter and initial quality across the alpine timberline ecotone in Western Sichuan, China. Chinese Journal of Applied Ecology, 26，3602-3610. [杨林, 邓长春, 陈亚梅, 和润 莲, 张健, 刘洋 (2015). 川西高山林线交错带调落叶分 解速率与初始质量的关系。应用生态学报, 26 , 3602-3610.]

Zackrisson O, DeLluca TH, Gentili F, Sellstedt A, Jaderlund A (2009). Nitrogen fixation in mixed Hylocomium splendens moss communities. Oecologia, 160, 309-319.

Zackrisson O, Deluca TH, Nilsson MC, Sellstedt A, Berglund LM (2004). Nitrogen fixation increases with successional age in boreal forests. Ecology, 85, 3327-3334.

Zhang Y, Guo LD (2007). Arbuscular mycorrhizal structure and fungi associated with mosses. Mycorrhiz, 17, 319325.

Zheng YP, Zhao JC, Zhang BC, Xu M, Liang HZ, Li M (2010). Influences of different physicochemical-chemical factors on three Cyanobacteria separated from biological soil crusts. Agriculture Research in the Arid Area, 28, 206211. [郑云普, 赵建成, 张丙昌, 徐明, 梁红柱, 李敏 (2010). 不同理化因子对荒漠生物结皮中三种蓝藻生长 的影响. 干旱地区农业研究, 28, 206-211.]

Zielke M, Ekker A, Olsen R, Spjelkavik S, Solheim B (2002). The influence of abiotic factors on biological nitrogen fixation in different types of vegetation in the high Arctic, Svalbard. Arctic Antarctic and Alpine Research, 34, 293 299.

Zielke M, Solheim B, Spjelkavik S (2005). Nitrogen fixation in the high arctic: Role of vegetation and environmental conditions. Arctic Antarctic and Alpine Research, 37, 372378.

责任编委: 程晓莉 责任编辑: 李 敏

www.plant-ecology.com 